\title{
Reducing radiation-induced gastrointestinal toxicity - the role of the PHD/HIF axis
}

\author{
Monica M. Olcina and Amato J. Giaccia \\ Department of Radiation Oncology, Division of Radiation and Cancer Biology, Stanford University, Stanford, California, USA.
}

\begin{abstract}
Radiotherapy is an effective treatment strategy for cancer, but a significant proportion of patients experience radiationinduced toxicity due to damage to normal tissue in the irradiation field. The use of chemical or biological approaches aimed at reducing or preventing normal tissue toxicity induced by radiotherapy is a long-held goal. Hypoxia-inducible factors (HIFs) regulate the production of factors that may protect several cellular compartments affected by radiation-induced toxicity. Pharmacological inhibitors of prolyl hydroxylase domain-containing enzymes (PHDs), which result in stabilization of HIFs, have recently been proposed as a new class of radioprotectors. In this review, radiation-induced toxicity in the gastrointestinal (GI) tract and the main cellular compartments studied in this context will be discussed. The effects of PHD inhibition on $\mathrm{GI}$ radioprotection will be described in detail.
\end{abstract}

\section{Introduction}

Approximately half of cancer patients will receive radiotherapy with either curative or palliative intent (1). Despite recent advances in radiotherapy treatment planning, normal tissue toxicity still limits the radiation dose that can be safely delivered (1). For example, when radiotherapy treatment is used to treat bladder or prostate cancer, it is often difficult to spare areas of the gastrointestinal (GI) tract, resulting in radiation-induced GI toxicity. Furthermore, patients with abdominal or head and neck tumors have a reasonable prognosis following treatment, making delayed toxic side effects a problem for a significant proportion of long-term survivors $(2,3)$.

Radiation results in detrimental cellular effects either through direct interaction of radiation with DNA or indirectly through the interaction of radiation with water and other tissue components. Indirect radiation effects result in the production of free radicals such as hydroxyl ( $\mathrm{HO} \cdot)$ and alkoxy $\left(\mathrm{RO}_{2} \cdot\right)$ radicals as well as reactive nitrogen species (4). Free radicals can react with DNA, resulting in DNA damage. Direct or indirect damage to DNA in the form of DNA breaks or replication stress results in the mounting of a DNA damage response (DDR), which includes p53 activation and cell cycle arrest, senescence, or apoptosis (5-9). A schematic of the sequence of events occurring following irradiation is shown in Figure 1.

The effects of radiation-induced normal tissue toxicity vary depending on the type of tissue being irradiated, the volume of tissue receiving irradiation, and the dose and dose rate delivered (3). Toxicity can result in symptoms ranging from mild or moderate to life threatening. In the most severe cases, symptoms may call for supportive treatment or changes to the radiotherapy treatment. Toxic effects are classified as acute, developing within days or weeks of radiation exposure, or as chronic, developing months or years

Conflict of interest: A.J. Giaccia holds stock in Fibrogen and is a founder and stockholder for RUGA Biotech.

Reference information: / Clin Invest. 2016;126(10):3708-3715. doi:10.1172/JCI84432. after treatment $(1,2)$. The majority of patients receiving radiation for the treatment of pelvic or intra-abdominal tumors experience acute radiation-induced GI toxicity symptoms (10). Furthermore, clinical and preclinical studies have shown that acute and chronic radiation-induced GI effects are not separate events, but are in fact linked, with some acute events playing a role in the development of late events (11-15). Late radiation-induced toxicity to the GI tract occurs from at least three months to several months or years after irradiation. Most intestinal compartments are affected by late radiation-induced effects, but damage to vascular and connective tissues is critical to this response (16). Chronic ulceration of the mucosa, mucosal atrophy, and fibrosis can underlie the induction of late toxicity effects. These events can lead to malabsorption, motility problems, and intestinal obstruction or perforation. Dysmotility can be especially problematic if it significantly alters the gut microbiome by increasing bacterial growth, resulting in further malabsorption and diarrhea $(17,18)$. Complications from radiation can result in the need for surgery or prolonged parenteral nutrition, which can have a negative effect on prognosis $(19,20)$. Additionally, a fatal syndrome (GI syndrome) involving diarrhea, bacterial translocation, and hemorrhage occurs when large areas of the intestine are irradiated (21). Thus, radiation has both short- and long-term effects that determine patient outcomes after treatment.

The effects of radiation-induced damage are complex since the GI tract, while lined with epithelial cells, also contains microvascular and nerve networks, as well as a variety of stromal and immune cells. The pathophysiology of radiation-induced toxicity reflects this complexity (3). Ideal pharmacological agents aimed at reducing radiation-induced toxicity should modulate the toxic effects of radiation on those cellular compartments. If these agents are to be used therapeutically in oncology, they should also be selective towards protection of sensitive normal tissue, but not the tumor. These agents should also allow feasible administration regimes and display a low-toxicity profile. Mitigators, administered after radiotherapy, can also be used in the event of accidental or other types of nonmedical exposures. Mitigators might be 


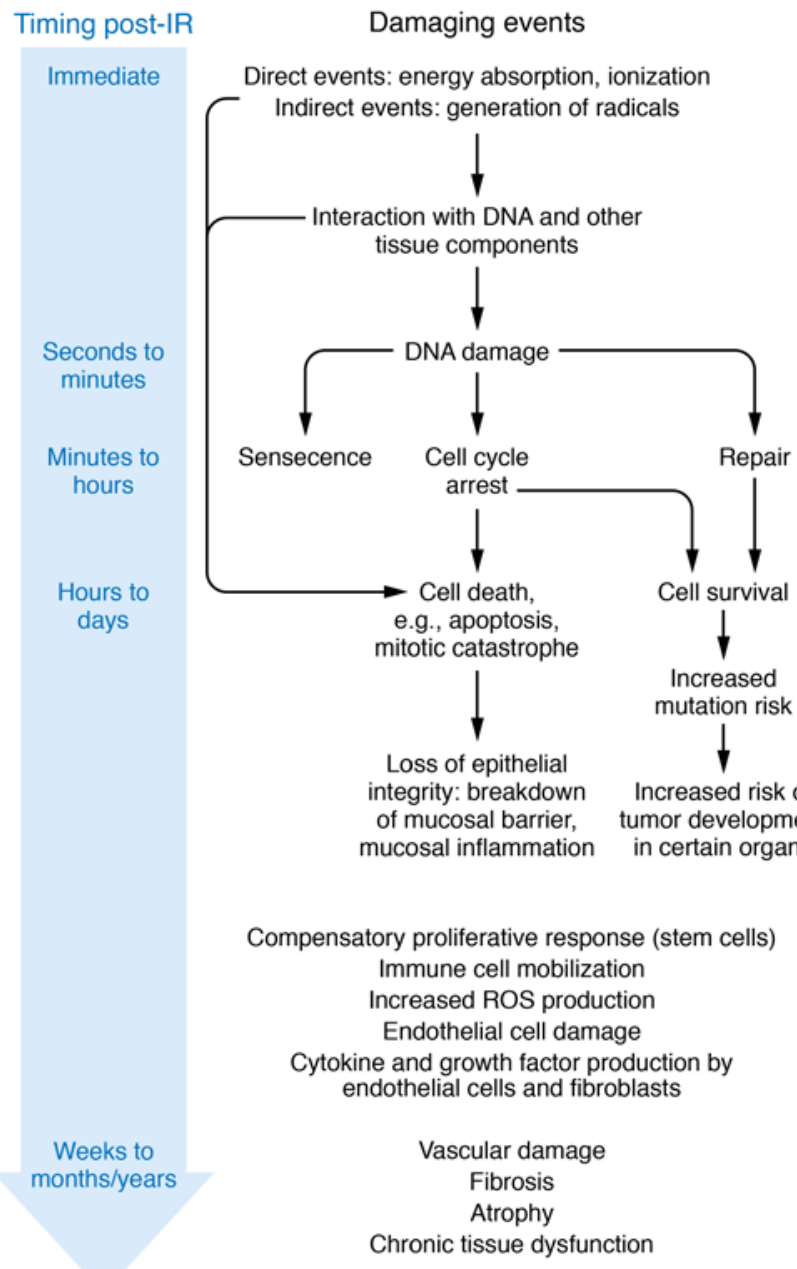

particularly useful if they are effective long after radiation exposure has occurred $(2,21)$.

The previously reported roles for HIF in promoting epithelial integrity, angiogenesis, and immune cell compartment mobilization led to the hypothesis that inducing HIF stability could reduce radiation-induced toxicity (22-26). Indeed, prolyl hydroxylase domain-containing enzyme (PHD) inhibitors, which stabilize HIF, have been recently described as a new class of agents that could reduce radiation-induced toxicity when given both before and up to 24 hours after irradiation in mice (27).

In this review, radiation-induced toxicity to the GI tract and the main cellular compartments studied in this organ will be described. A special focus will be placed on the role of PHD inhibitors in reducing radiation-induced toxicity of the GI tract.

\section{Pathophysiology of radiation-induced intestinal toxicity}

The intestinal epithelial lining is one of the most rapidly renewing system in the human body, with intestinal stem cells in the crypts mediating regeneration of the small intestine and colon (28, 29). Epithelial cells migrate along the villi before being shed into the lumen. It takes around six to eight days for cells to move from the crypt to the tip of the villus, and two to three days from the time of entry into the villus base until shedding from the tip $(28,29)$.
Figure 1. The sequence of damaging events occurring following irradiation. Damaging effects of irradiation on various cellular compartments can occur within $10^{-17}-10^{-13}$ seconds to months or years after irradiation, resulting in a variety of acute or chronic effects. A selection of these damaging effects and their consequences is shown to the right-hand side of the timeline. While the sequence of early events (within hours of irradiation) has been studied in detail, the timing of and relationships between events occurring weeks to months or years after irradiation are more complicated and are still incompletely understood. This complexity is reflected by a lack of arrows between events. Figure adapted from ref. 21.

Interestingly, radiation doses above $1 \mathrm{~Gy}$ can damage the intestinal mucosa, making GI toxic effects common in patients treated with abdominal radiotherapy. Epithelial cells in the crypt undergo early p53-dependent apoptosis within hours after irradiation, leading to shrinkage of crypts $(5,30)$. As a compensatory mechanism, the surviving cells hyperproliferate, leading to a temporary increase in crypt size (31). Damage to the intestinal epithelium results from inadequate replacement of the surface epithelium following apoptotic and mitotic death in the crypt (32). Intestinal mucositis symptoms occur once the epithelial barrier breaks down, allowing for fluid loss and bacterial entry (32). The more extensive apoptosis that occurs at high radiation doses prevents intestinal repopulation and results in GI failure (32).

Endothelial cells are also thought to be involved in the normal tissue response to radiation because apoptosis of this cell type can result in the release of growth factors, chemokines, and cytokines that mediate inflammatory, thrombotic, and antifibrinolytic responses (33). Part of this inflammatory response stimulates macrophages, which may act as effective sensors of such damage (3). Once activated, macrophages secrete a number of chemokines and cytokines that facilitate neutrophil recruitment. Neutrophils that infiltrate the damaged tissue can also release factors that lead to further circulating monocyte recruitment, highlighting the crosstalk between cell types. In addition to responding to the initial damage by mounting an inflammatory response, macrophages also help resolve tissue damage by facilitating the removal of apoptotic bacteria, cellular debris, and neutrophils $(3,34)$. The role of neutrophils in responding to radiation-induced GI toxicity is not fully understood. On one hand, ROS produced by neutrophils are likely important in the response to inflammation and infection following breakdown of the mucosal and vascular barriers (3). On the other hand, chronic generation of ROS may contribute to the late effects of radiation on normal tissue such as a fibro-atrophic process (35). The different cellular compartments affected by radiation-induced toxicity in the GI tract are shown in Figure 2.

\section{$\mathrm{HIF/PHD} \mathrm{biology} \mathrm{and} \mathrm{strategies} \mathrm{used}$ to modulate PHD activity/HIF stability}

HIFs are critical transcription factors regulating physiological and pathological processes that occur as oxygen tension decreases in tissues, including in organs where hypoxic gradients naturally occur such as the GI tract or bone marrow $(36,37)$. There are three main HIF- $\alpha$ isoforms (HIF-1 $\alpha, 2 \alpha$, and $3 \alpha$ ), and their expression and importance is tissue dependent (38).

HIF- $1 \alpha$ and HIF- $2 \alpha$ form transcriptionally active heterodimers with HIF-1 $1 \beta$. HIF- $\alpha$ protein stability is primarily regulated by a 


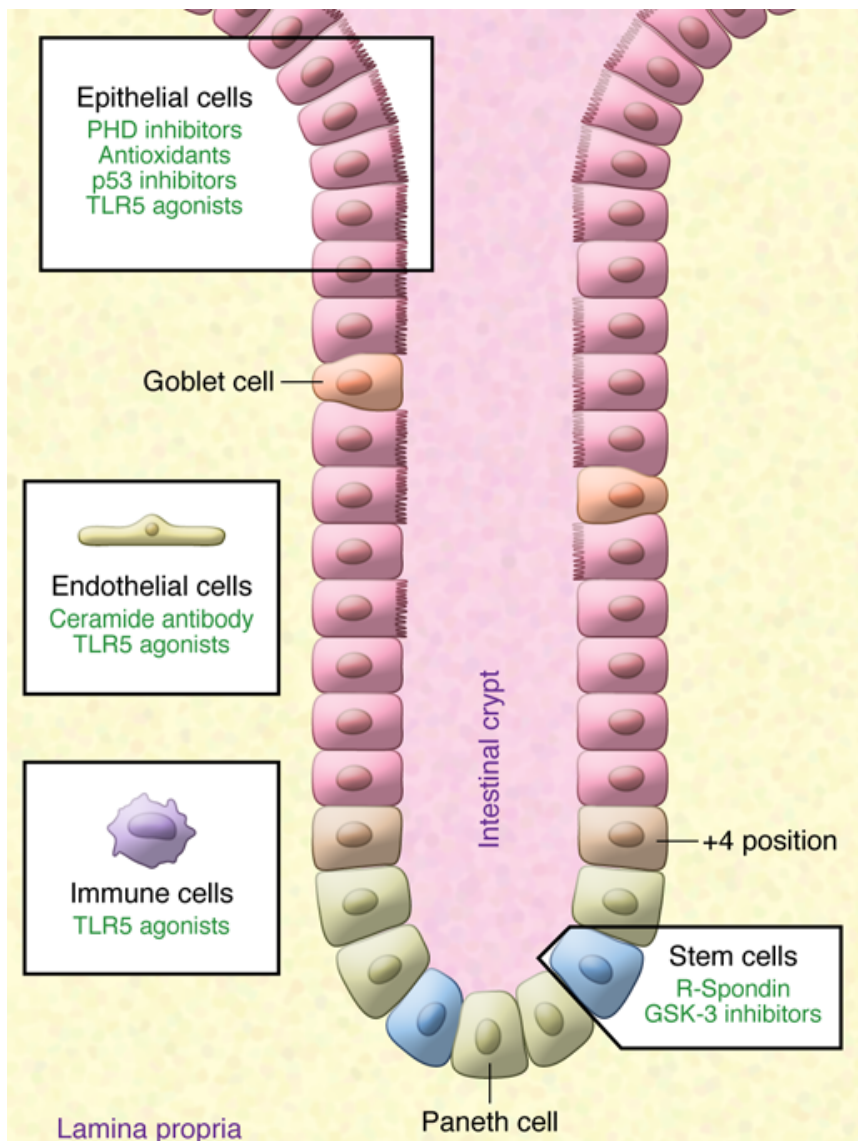

Lamina propria

Paneth cell

Figure 2. Cellular compartments affected by radiation-induced toxicity in the Gl tract. Epithelial (drawn in red), endothelial (drawn in light blue), immune (drawn in purple) as well as stem cells (LGR5 ${ }^{+}$and $\mathrm{BMI1}{ }^{+}$cells) (drawn in dark blue) can all be damaged following irradiation of the GI tract and can contribute to radiation-induced toxicity. The green text within the boxes represents the chemical and biological strategies that may protect against damage to the cellular compartments they lie adjacent to.

family of PHD-containing proteins (38). Enzymatically, PHDs are nonheme iron-containing 2-oxoglutarate-dependent oxygenases (39). When oxygen is present, PHD proteins can hydroxylate two proline residues near the $\mathrm{N}$-terminal transactivation domain of HIF- $\alpha(40,41)$. Hydroxylation allows binding to the von HippelLindau (VHL) ubiquitin ligase, which facilitates proteasomal degradation of HIF- $\alpha$ proteins. In the absence of oxygen, PHDs have a diminished ability to hydroxylate HIFs, leading to the accumulation of HIF- $\alpha$ protein $(38,41)$.

Pharmacological stabilization of HIF-1 can be achieved by the use of a number of compounds. The so-called hypoxia mimetics cobalt chloride $\left(\mathrm{CoCl}_{2}\right)$ and the iron chelator desferrioxamine can stabilize HIF-1 $(42,43)$. Similarly, the organomercurial compound mersalyl and the putative PHD2 inhibitor baicalein have been proposed to reduce HIF ubiquitination, thereby increasing its stability $(44,45)$. Interestingly, the anesthetic isoflurane can also induce HIF-1-dependent gene expression by upregulating HIF-1 $\alpha$ (46).

More recently, two different PHD inhibitors, FG-4497 and dimethyloxallyl glycine (DMOG), have been shown to result in mucosal protection in murine models of GI damage $(26,27)$. FG-4497 was shown to protect the intestinal mucosa in models of chemical-induced colitis, while DMOG protects against radiation-induced toxicity $(26,27)$. Both of these PHD inhibitors were shown to stabilize HIFs, resulting in the activation of downstream HIF target genes $(26,27)$.

\section{PHD/HIF and gut epithelium protection}

The monolayer of epithelial cells lining the colon is capable of secreting mucus and is organized into microvilli and apical tight junctions. These features allow the epithelial layer to serve an important barrier function $(10,26)$. HIF can promote epithelial integrity through the regulation of genes such as intestinal trefoil factor (ITF) and ecto-5'-nucleotidase (CD73). Consequently, pharmacological and genetic approaches (conditional deletion of HIF-1 $\alpha$ in epithelial cells) have demonstrated a protective role for HIF- $1 \alpha$ in a model of colitis in mice (26). Interestingly, HIF$1 \alpha$ expression appears to be elevated in patients with ulcerative colitis, Crohn's disease, and ischemic colitis (47-49). The role of PHDs in models of colitis is described in further detail in a separate article in this issue and will not be described in further detail here. Instead, a detailed account of the role of PHDs in radiationinduced toxicity will be described below.

PHD inhibition and epithelial integrity in radiotherapy. The effects of irradiation alone on HIF stabilization in normal tissues appear complex, with HIF- $1 \alpha$ and HIF- $2 \alpha$ being detected in the colon, liver, and kidneys, but not in other organs such as the lung following irradiation (27). When the small molecule DMOG was used in combination with irradiation, HIF- $1 \alpha$ and HIF- $2 \alpha$ stabilization in the GI tract was significantly increased following DMOG and irradiation compared to irradiation alone (27). Interestingly, GI tract-specific genetic knockdown of all three PHDs was necessary for abundant HIF expression, while knockdown of individual PHDs had minimal effects. Most importantly, triple PHD knockout mice exhibited dramatically improved survival after $18 \mathrm{~Gy}$ of total abdominal irradiation (TAI), with $70 \%$ of knockout mice surviving after 30 days of irradiation. Treatment of mice with DMOG was shown to result in stabilization of HIF- $1 \alpha$ and HIF- $2 \alpha$ in both the small intestine and colon, and this stabilization was also correlated with improved survival after TAI (27). Furthermore, microcolony crypt survival assays demonstrated a 4- and 22-fold improvement in survival after 18 and 20 Gy TAI, respectively, in the group treated with DMOG compared to the group treated with saline. These data suggest that PHD inhibition can reduce crypt death or increase regeneration following irradiation. Measurement of apoptosis revealed reduced staining in the colon and intestine in the DMOG-treated group. Interestingly, this decrease in apoptosis did not correlate with decreased histone H2AX phosphorylation at serine 139 ( $\gamma \mathrm{H} 2 \mathrm{AX})$, suggesting that the mechanism behind this response does not involve changes in DDR signaling (27). Expression of the HIF target genes ITF and multidrug resistance protein 1 (MDR1) was also increased in the jejunum of DMOG-treated animals. Importantly, DMOG-treated mice appeared to live with minimal associated morbidity for 20 months following irradiation. Although mice were smaller than unirradiated controls, they did not develop any malignancies, fistulas, or palpable fibrosis (27). Figure 3 shows 


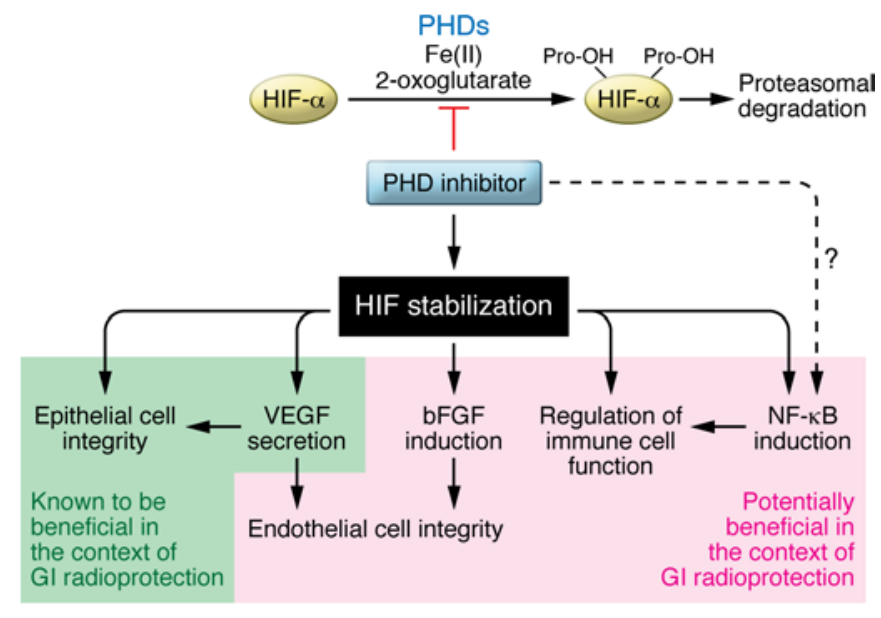

Figure 3. PHD inhibition induces responses that may result in normal tissue radioprotection in the GI tract. PHD inhibition will result in HIF- $\alpha$ stabilization due to decreased HIF hydroxylation and consequently less degradation (40, 41). Once stabilized, HIF- $\alpha$ can mediate gene expression changes that can contribute to maintaining epithelial barrier integrity following irradiation (27). Protective effects of PHD inhibitors also appear to be dependent on HIF-2 $\alpha$-induced increases in VEGF expression, which correlate with increased microvasculature (27). HIF- $\alpha$ stabilization can also result in bFGF induction, regulation of immune cell function, and NF- $\mathrm{KB}$ activation $(22,66,96)$. These effects are represented by solid arrows. The $\mathrm{NF}-\mathrm{KB}$ inducer, IKK $\beta$, has a conserved PHD1 hydroxylation site, suggesting that NF- $\kappa B$ can also be regulated by PHD inhibition (97). Dotted arrow and pink background represent those effects that would be predicted to be mediated by either HIF- $\alpha$ stabilization or directly as a result of PHD inhibition and could result in radioprotection against radiation-induced $\mathrm{GI}$ toxicity. These effects have not been formally shown to occur in the context of radioprotection to date. Effects within the green background have been shown to contribute to protection against radiation-induced GI toxicity.

how the effects of PHD inhibition may result in reduced radiation-induced toxicity in the GI tract.

Targeting PHDs and 553 to improve epithelial integrity following irradiation. As mentioned above, irradiation results in p53-dependent apoptosis of intestinal epithelial cells $(5,30)$. The importance of p53 in modulating survival following irradiation is supported by a study in which p53-deficient mice survived higher radiation doses (whole body) than their littermate controls. Importantly, inhibition of p53 with the small molecule pifithrin- $\alpha$ was proposed to limit radiation-induced apoptosis in normal tissues without compromising the radiosensitivity of the tumor xenografts. Targeting p53 with inhibitors such as pifithrin- $\alpha$ is not predicted to increase the chances of developing future tumors since altering the p53-mediated acute response to DNA damage does not appear to alter its tumor suppressor functions (50-53). At a mechanistic level, p53 inhibition by pifithrin- $\alpha$ appeared to reduce p53-induced DNA replication arrest in tissues with high proliferation rates following whole-body irradiation. The increased DNA replication observed in mice treated with the inhibitor correlated with reduced levels of intestinal epithelial cell apoptosis. In contrast, a subsequent study showed that p53 deficiency sensitizes mice to higher doses of radiation associated with the development of GI syndrome (50). p53 deficiency in this context was associated with enhanced death of damaged cells in the GI epithelium. Given that p21-deficient mice were also more sensitive to radiation-induced GI syndrome, the authors proposed that $\mathrm{p} 53$ / p21-mediated cell cycle arrest could have a protective role in epithelial radiation-induced toxicity in the GI tract (54). Subsequent studies have further challenged the importance of p53-dependent apoptosis specifically in radiation-induced GI toxicity by showing that deletion of the proapoptotic genes Bax and Bak1 from GI epithelial or endothelial cells does not protect against radiationinduced GI syndrome. However, deletion of p 53 from GI epithelial cells but not endothelial cells was shown to result in sensitization to GI syndrome in mice, supporting the concept that 553 functions to protect against radiation-induced GI syndrome but may do so through apoptosis-independent mechanisms (55).

As described above, the radioprotective effects conferred by DMOG appear to be independent of changes in the DDR. The combination of DMOG (or other PHD inhibitors) with agents affecting the response to radiation-induced DNA damage, such as p53 inhibitors, could perhaps result in increased synergistic radioprotective effects. Moreover, the HIF-1 $\alpha$ oxygen-dependent degradation (ODD) domain can bind to a p53 dimer, further supporting the potential crosstalk between these two major transcription factors (56). Combining PHD inhibitors with therapeutic efforts aimed at targeting p53 transcriptional targets might be another option that could be considered to improve normal tissue radioprotection (57-59). Recent studies have demonstrated that the p53 transcriptional target, p53-upregulated modulator of apoptosis (PUMA), mediates radiation-induced intestinal cell apoptosis, while p21-dependent prevention of persistent DNA damage has been suggested to facilitate regeneration (58-60). The use of the glycogen synthase kinase-3 (GSK-3) inhibitors, CHIR99021, has thus been proposed for radioprotection since CHIR99021 treatment decreased PUMA induction as well as p53 acetylation at K120, without affecting p21 induction or p53 phosphorylation (or stability) following irradiation. These effects correlated with decreased apoptosis of leucine-rich repeat-containing GPCR $5^{+}$ $\left(\mathrm{LGR}^{+}\right)$cells in both crypt regeneration assays in vitro and in mice (59). The combination of PHD inhibitors with GSK-3 inhibitors such as CHIR99021 could be an interesting future area of study.

\section{Microvascular injury in radiation-induced toxicity} Radiation can damage blood vessels, contributing to late radiation-induced toxicity due to changes in endothelial cell physiology. These changes include endothelial cell apoptosis, detachment from the basement membrane, and increased fibrin deposition $(10,61)$. However, the role of microvascular injury in acute toxicity effects is under debate (10).

Paris and colleagues suggested that radiation-induced damage to stem cell populations was a result of microvascular injury occurring before epithelial cell damage. This response was proposed to be governed by the ceramide pathway based on studies using acid sphingomyelinase-deficient mice, which fail to generate the proapoptotic lipid ceramide in the endothelium following irradiation (62). Administration of bFGF reduced the levels of endothelial apoptosis, suggesting that the sensitivity of the microvasculature to radiation-induced toxicity may be dependent on bFGF levels. Indeed, despite the ubiquitous expression of bFGF in large vessels, basement membranes within the microvasculature have very low levels of bFGF. These findings are consistent with 
the observation that out of all the vascular structures, capillaries are the most sensitive to radiation-induced damage (62). Despite these observations, the importance of endothelial cell apoptosis remains controversial (10)

It is important to note that endothelial-specific overexpression of HIF- $1 \alpha$ or HIF- $2 \alpha$ did not improve survival after 18 Gy with respect to littermate controls (27). Instead, HIF-2 $\alpha$ expression in epithelial cells was sufficient to improve survival after 18 Gy TAI, resulting in increased VEGF expression in the GI epithelia and serum. DMOG treatment also led to increased VEGF expression and VEGF serum levels in the jejunum and colon, and these increases appeared to correlate with increased microvessel density following irradiation in the crypts of the jejunum (27). Moreover, DMOG-induced radioprotection appears to be partly dependent on VEGF since inhibition of VEGF function abrogated the protective effects of DMOG. These results suggest that HIF$2 \alpha$ expression in the epithelial cells but not the endothelial cells is important for radioprotection and that these effects may be mediated by increased HIF-2 $\alpha$-induced expression of VEGF. (27). The effects of DMOG also appear to mitigate radiation-induced damage when administered 24 hours after irradiation, suggesting that this compound could also be used as a medical countermeasure to radiation exposure. Again, these effects appeared to be dependent, in part, on VEGF (27). The requirement for HIF-2 $\alpha$ for protection against radiation-induced GI toxicity contrasts with the requirement for HIF-1 $\alpha$ in protection against colitis $(26,27)$.

\section{The role of inflammation in radiation-induced toxicity}

Radiation-induced damage leads to a robust inflammatory response. Given that inflamed tissues are often hypoxic, it is perhaps not surprising that HIFs and PHDs have been reported to have a regulatory effect on the inflammatory response in a number of pathological situations. For example, HIF-1 $\alpha$ transcriptionally regulates a number of glycolytic pathway enzymes that allow macrophages and neutrophils to carry out glycolysis under the hypoxic conditions that characterize inflamed tissues $(22,36)$. HIF-1 may also regulate leukocyte trafficking through upregulation of CD73 as well as neutrophil recruitment through HIFdependent CD55 induction $(24,25,63)$.

The transcription factor $\mathrm{NF}-\kappa \mathrm{B}$ provides an additional link between HIF and neutrophils. NF- $\mathrm{BB}$ can orchestrate innate and adaptive immune responses to infectious agents (64). NF- $\kappa \mathrm{B}$ activation by HIF- $1 \alpha$ can prevent neutrophil apoptosis in inflammatory hypoxic conditions, an effect that could be exploited to combat the inflammatory response induced by irradiation. Furthermore, the NF- $\kappa \mathrm{B}$ inducer IKK $\beta$ has a conserved PHD1 hydroxylation site, suggesting that NF- $\kappa \mathrm{B}$ can also be regulated by PHD1. The crosstalk between HIF- $1 \alpha$ and NF- $\kappa \mathrm{B}$ is further complicated by the fact that NF- $\kappa \mathrm{B}$ can itself induce HIF- $1 \alpha$ expression, presumably through IKK $\beta$. Together, these data suggest that PHD inhibition might also result in regulation of $\mathrm{NF}-\kappa \mathrm{B}$, which could prove beneficial in the context of normal tissue radioprotection $(65,66)$.

Importantly, NF- $\mathrm{KB}$ signaling can blunt p53 activity as well as induce ROS scavengers, cytokines, and apoptosis inhibitors, all of which could contribute to reduced radiation-induced toxicity $(67,68)$. Indeed, mice with increased GI radiosensitivity exhibit defects in NF- $\kappa$ B signaling, presumably due to increased intestinal crypt apoptosis. NF- $\kappa \mathrm{B}$ can be activated by the induction of Toll-like receptors (TLRs) (69). TLRs recognize commensal microflora, a function that appears important in both maintaining GI homeostasis and protecting against injury (70). Burdelya et al. investigated the factors produced by human gut microorganisms that bind TLRs to activate NF- $\mathrm{B}$. This approach led them to engineer an $\mathrm{NF}-\kappa \mathrm{B}$ activator (CBLB502) that included the complete $\mathrm{N}$ - and C-terminal domains of flagellin, the only known natural ligand of TLR5 $(71,72)$. The number of apoptotic cells in the small intestine of irradiated mice was reduced when CBLB502 was administered 30 minutes before $15 \mathrm{~Gy}$ of total body irradiation. CBLB502 administration also reduced endothelial cell apoptosis, which was positively correlated with maintenance of intestinal crypt size and cell density in the treated group compared to the control group. Importantly, administration of CBLB502 did not reduce tumor radiosensitivity in two subcutaneous tumor models and did not increase radiation-induced tumorigenesis (72).

\section{The role of intestinal stem cells in radiation- induced toxicity}

As mentioned previously, death of rapidly proliferating intestinal progenitor cells following irradiation leads to inadequate villus epithelium replacement. Intestinal stem cells, which express LGR5 or the polycomb complex protein BMI1, are reported to facilitate regeneration after radiation-induced damage (10). LGR5 ${ }^{+}$cells are interspersed between Paneth cells and can be expressed throughout the intestine, while $\mathrm{BMI}^{+}$cells are found at the bottom of crypts and predominantly at position +4 (four cells above the base) in the proximal small intestine crypts $(73,74)$. While mouse LGR5 ${ }^{+}$ cells are mitotically active, $\mathrm{BMI} 1^{+}$cells are quiescent and considered more radioresistant than $\mathrm{LGR}^{+}$cells. Moreover, $\mathrm{BMI}^{+}$cells are capable of rapid proliferation and mobilization after injury to facilitate regeneration (75). However, a recent study suggested that only LGR5 ${ }^{+}$cells are required for regeneration (76).

HIFs could directly affect the function or induce factors that affect the function of these intestinal stem cells $(27,77)$; therefore, targeting PHDs could potentially reduce radiation-induced damage through the regenerative effects of HIFs on these stem cells. Importantly, expression of HIFs in $\mathrm{LGR}^{+}$and $\mathrm{BMI} 1^{+}$cells does not provide radioprotection on its own. Only HIF expression in epithelial cells afforded radioprotection, suggesting that endothelial and intestinal stem cells may work together with epithelial cells to mediate radioprotection (27).

\section{HIF and tumor radiation response}

The mechanisms through which irradiation regulates HIF expression and the consequence of such regulation for the tumor radiation response are complex $(78,79)$. The PI3K/Akt/mTOR pathway, for example, has been reported to increase protein stability of HIF- $1 \alpha$ in lung cancer cells. Interestingly, in radioresistant lung cancer cells an increased interaction between Hsp90 and HIF-1 $\alpha$ may facilitate increased HIF-1 $\alpha$ stability following irradiation. Disruption of this interaction with the use of an Hsp90 inhibitor resulted in decreased HIF-1 $\alpha$ levels, decreased angiogenic potential, and increased sensitivity of these cells to irradiation both in vitro and in vivo (80). 
Radiation-induced reoxygenation has been proposed to increase HIF stabilization in the nucleus as well as to increase translation of HIF target genes (81). These effects were proposed to increase cytokines responsible for protecting endothelial cells from radiation-induced apoptosis. The increased vascular damage observed following HIF-1 inhibition was proposed to be responsible for increased tumor radiosensitivity (81). A recent study supported the idea of increased HIF-1 $\alpha$ stabilization following radiation-induced reoxygenation by demonstrating that tumor cells that survived irradiation had increased HIF-1 $\alpha$ expression in regions undergoing reoxygenation, allowing these cells to move towards tumor blood vessels (82). HIF-1 inhibition resulted in decreased movement of surviving tumor cells towards the vessels, coupled with a decrease in tumor recurrence following radiotherapy (82). Loss of HIF-1 $\alpha$ has also been associated with increased radiosensitivity of cancer cells and tumor xenografts in a number of studies $(79,83)$. Interestingly, Williams and colleagues suggested that the effects of hypoxia on tumor radiation response were not simply governed by the effects of reduced oxygen on radiation-induced radical formation, but that radiation resistance in hypoxic regions was likely also a result of HIF-1-dependent gene expression changes (83). Supporting this notion, both the size of the hypoxic fraction of a tumor and the expression of HIF- $1 \alpha$ have been correlated with poor prognosis following radiotherapy (84-86). In contrast, HIF-1 also has been found to enhance apoptosis, decrease proliferation, and regulate metabolism following irradiation such that loss of HIF-1 may promote radioresistance in certain situations. The conclusion from this study appeared to be that the timing of radiation as well as the microenvironment of each specific tumor should be taken into careful consideration when targeting HIF-1 together with radiation therapy (78).

While there is a large body of literature examining the complex role of HIF-1 $\alpha$ in tumor radiosensitization, much less is know about the effects of HIF- $2 \alpha$ on this response. HIF- $2 \alpha$ deficiency has been reported to induce p53 activity and increase cell death and radiation sensitivity in vitro (87). Given that many GI radioprotective effects are dependent on HIF- $2 \alpha$, it would be of interest to investigate the effects of HIF- $2 \alpha$ expression on tumor radiation response in further detail.

HIF stabilization following PHD inhibition with DMOG has not been shown to result in significant tumor radioprotective effects in xenograft tumor models (27). It is tempting to speculate that acute HIF stabilization following pharmacological PHD inhibition is insufficient to increase tumor radioresistance. The long-term effects of PHD inhibition on patient prognosis following radiation therapy should be investigated in detail if this pharmacological approach is to be used clinically for normal tissue radioprotection. For example, the effects of HIF activation on future tumor development should be carefully studied given the previously established associations between HIF-1 $\alpha$ and/or HIF$2 \alpha$ expression with increased vascularization and poor prognosis $(88,89)$. Interestingly, loss of PHD2 with subsequent HIF stabilization normalizes blood vessels, leading to enhanced delivery of chemotherapeutic drugs and decreased primary tumor size and metastasis $(90,91)$. Reorganization of blood vessels by PHD loss might also be expected to increase tumor sensitivity to irradiation.

\section{Conclusions}

Radiotherapy is a very effective cancer treatment strategy; however, a significant proportion of patients will experience radiationinduced toxicity due to damage to normal tissue in the irradiation field. The need to irradiate normal tissue margins containing microscopic disease can result in toxic side effects with a negative impact on quality of life and treatment outcome. The use of chemical and biological approaches to reduce or prevent this damage has been proposed as a strategy for improving radiation treatment (21). Despite interesting research being carried out in this area, it has been difficult to translate these important findings into the clinic. One of the problems with some preclinical studies performed to date is the use of high and frequently single doses of radiation that do not necessarily recapitulate the radiation schedules used clinically in fractionated radiotherapy. These single fractions used preclinically often target large GI volumes, perhaps making these better models for acute GI syndrome rather than the intestinal radiation toxicity (enteropathy) observed in radiotherapy patients (10). More research is also needed in order to fully understand the importance of clonogenic cell death versus intestinal crypt apoptosis in the radiation response of GI cells and the relationship of these responses to the clinical situation. Concerns over the potential tumor radiation protection of some of the agents should also be addressed more rigorously. Most preclinical studies have attempted to address these concerns at least in xenograft models $(92,93)$.

The central role of the PHD/HIF axis in critical processes involved in the normal tissue radiation response highlights the potential of these inhibitors as radioprotectors and/or mitigators. PHD inhibitors such as FG-4592, DS-1093a, GSK1278863, and AKB-6548 are already in clinical trials for the treatment of anemia in patients with chronic kidney disease (94). This may facilitate their use as mitigators after large-scale accidental radiation incidents or as part of radiotherapy treatment protocols in the future (27). As our understanding of immune cell function in normal tissue responses to irradiation increases, it is likely that therapeutic approaches aimed at targeting this cellular compartment will become important. Modulation of HIF stability by PHD inhibition also will likely play a role in the regulation of these immune cell functions.

The ability to protect normal tissue from radiation-induced damage could one day allow the use of radiation as a 'systemic' therapy rather than as a treatment that is mainly used for locoregional control. The potential to realize this goal is so enticing that it warrants further research in this exciting field (95).

\section{Acknowledgments}

We would like to apologize to the authors of all the excellent work that we were not able to reference in this study due to space limitations. This work was supported by the NIH Grants CA-67166, CA-198291, and CA-197713, the Silicon Valley Foundation, the Sydney Frank Foundation, and the Kimmelman Fund (all to AJG). MMO is a Cancer Research Institute Irvington Fellow supported by the Cancer Research Institute.

Address correspondence to: Amato J. Giaccia, Stanford University, CCSR-South, Room 1255, 269 Campus Drive, Stanford, California 94305-5152, USA. Phone: 650.723.7366; E-mail: giaccia@ stanford.edu. 
1. Kerns SL, et al. The prediction of radiotherapy toxicity using single nucleotide polymorphismbased models: a step toward prevention. Semin Radiat Oncol. 2015;25(4):281-291.

2. Bentzen SM. Preventing or reducing late side effects of radiation therapy: radiobiology meets molecular pathology. Nat Rev Cancer. 2006;6(9):702-713.

3. François A, Milliat F, Guipaud O, Benderitter M. Inflammation and immunity in radiation damage to the gut mucosa. Biomed Res Int 2013;2013:123241.

4. Desouky O, Ding N, Zhou G. Targeted and nontargeted effects of ionizing radiation.J Radiat Res Appl Sci. 2015;8(2):247-254.

5. Merritt AJ, et al. The role of p53 in spontaneous and radiation-induced apoptosis in the gastrointestinal tract of normal and p53-deficient mice. Cancer Res. 1994;54(3):614-617.

6. Kastan MB, Onyekwere O, Sidransky D, Vogelstein B, Craig RW. Participation of $\mathrm{p} 53$ protein in the cellular response to DNA damage. Cancer Res. 1991;51(23 Pt 1):6304-6311.

7. Canman CE, et al. Activation of the ATM kinase by ionizing radiation and phosphorylation of $\mathrm{p} 53$ Science. 1998;281(5383):1677-1679.

8. Meyer $\mathrm{F}$, et al. Interaction between antioxidant vitamin supplementation and cigarette smoking during radiation therapy in relation to long-term effects on recurrence and mortality: a randomized trial among head and neck cancer patients. Int J Cancer. 2008;122(7):1679-1683.

9. Lomax ME, Folkes LK, O'Neill P. Biological consequences of radiation-induced DNA damage: relevance to radiotherapy. Clin Oncol ( $R$ Coll Radiol). 2013;25(10):578-585.

10. Hauer-Jensen M, Denham JW, Andreyev HJ. Radiation enteropathy--pathogenesis, treatment and prevention. Nat Rev Gastroenterol Hepatol. 2014;11(8):470-479.

11. Osborne JW, Prasad KN, Zimmerman GR. Changes in the rat intestine after $\mathrm{x}$-irradiation of exteriorized short segments of ileum. Radiat Res. 1970;43(1):131-142.

12. Bourne RG, Kearsley JH, Grove WD, Roberts SJ. The relationship between early and late gastrointestinal complications of radiation therapy for carcinoma of the cervix. Int J Radiat Oncol Biol Phys. 1983;9(10):1445-1450.

13. Wang CJ, et al. The correlation of acute toxicity and late rectal injury in radiotherapy for cervical carcinoma: evidence suggestive of consequential late effect (CQLE). Int J Radiat Oncol Biol Phys. 1998;40(1):85-91.

14. Denham JW, Hauer-Jensen M, Kron T, Langberg CW. Treatment-time-dependence models of early and delayed radiation injury in rat small intestine. Int J Radiat Oncol Biol Phys. 2000;48(3):871-887.

15. Fellin G, et al. Long term rectal function after high-dose prostatecancer radiotherapy: results from a prospective cohort study. Radiother Oncol. 2014;110(2):272-277.

16. Denham JW, Hauer-Jensen M. The radiotherapeutic injury--a complex 'wound'. Radiother Oncol. 2002;63(2):129-145.

17. Husebye E, Hauer-Jensen M, Kjørstad K, Skar V. Severe late radiation enteropathy is characterized by impaired motility of proximal small intestine.
Dig Dis Sci. 1994;39(11):2341-2349.

18. Husebye E, Skar V, Høverstad T, Iversen T, Melby K. Abnormal intestinal motor patterns explain enteric colonization with gram-negative bacilli in late radiation enteropathy. Gastroenterology. 1995;109(4):1078-1089.

19. Galland RB, Spencer J. The natural history of clinically established radiation enteritis. Lancet. 1985;1(8440):1257-1258.

20. Silvain C, et al. Long-term outcome of severe radiation enteritis treated by total parenteral nutrition. Dig Dis Sci. 1992;37(7):1065-1071.

21. Citrin D, Cotrim AP, Hyodo F, Baum BJ, Krishna MC, Mitchell JB. Radioprotectors and mitigators of radiation-induced normal tissue injury. Oncologist. 2010;15(4):360-371.

22. Cramer T, et al. HIF-1alpha is essential for myeloid cell-mediated inflammation. Cell. 2003;112(5):645-657.

23. Krock BL, Skuli N, Simon MC. Hypoxia-induced angiogenesis: good and evil. Genes Cancer. 2011;2(12):1117-1133.

24. Synnestvedt K, et al. Ecto-5'-nucleotidase (CD73) regulation by hypoxia-inducible factor- 1 mediates permeability changes in intestinal epithelia. J Clin Invest. 2002;110(7):993-1002.

25. Thompson LF, et al. Crucial role for ecto-5'nucleotidase (CD73) in vascular leakage during hypoxia. JExp Med. 2004;200(11):1395-1405.

26. Robinson A, Keely S, Karhausen J, Gerich ME, Furuta GT, Colgan SP. Mucosal protection by hypoxia-inducible factor prolyl hydroxylase inhibition. Gastroenterology. 2008;134(1):145-155.

27. Taniguchi CM, et al. PHD inhibition mitigates and protects against radiation-induced gastrointestinal toxicity via HIF2. Sci Transl Med. 2014;6(236):236ra64

28. Potten CS, Taylor Y, Hendry JH. The doubling time of regenerating clonogenic cells in the crypts of the irradiated mouse small intestine. Int J Radiat Biol. 1988;54(6):1041-1051.

29. van der Flier LG, Clevers H. Stem cells, selfrenewal, and differentiation in the intestinal epithelium. Annu Rev Physiol. 2009;71:241-260.

30. Inagaki-Ohara K, et al. p53-dependent radiationinduced crypt intestinal epithelial cells apoptosis is mediated in part through TNF-TNFR1 system. Oncogene. 2001;20(7):812-818.

31. Withers HR, Elkind MM. Microcolony survival assay for cells of mouse intestinal mucosa exposed to radiation. Int J Radiat Biol Relat Stud Phys Chem Med. 1970;17(3):261-267.

32. Erickson BA, Otterson MF, Moulder JE, Sarna SK. Altered motility causes the early gastrointestinal toxicity of irradiation. Int J Radiat Oncol Biol Phys. 1994;28(4):905-912.

33. Wang J, Boerma M, Fu Q, Hauer-Jensen M. Significance of endothelial dysfunction in the pathogenesis of early and delayed radiation enteropathy. World J Gastroenterol. 2007;13(22):3047-3055.

34. Soehnlein O, Lindbom L. Phagocyte partnership during the onset and resolution of inflammation. Nat Rev Immunol. 2010;10(6):427-439.

35. Robbins ME, Zhao W. Chronic oxidative stress and radiation-induced late normal tissue injury: a review. Int J Radiat Biol. 2004;80(4):251-259.

36. Fraisl P, Aragonés J, Carmeliet P. Inhibition of oxygen sensors as a therapeutic strategy for ischaemic and inflammatory disease. Nat Rev Drug Discov. 2009;8(2):139-152.

37. Eltzschig HK, Bratton DL, Colgan SP. Targeting hypoxia signalling for the treatment of ischaemic and inflammatory diseases. Nat Rev Drug Discov. 2014;13(11):852-869.

38. Masson N, Ratcliffe PJ. Hypoxia signaling pathways in cancer metabolism: the importance of co-selecting interconnected physiological pathways. Cancer Metab. 2014;2(1):3.

39. Schofield CJ, Ratcliffe PJ. Oxygen sensing by HIF hydroxylases. Nat Rev Mol Cell Biol. 2004;5(5):343-354.

40. Chan DA, Sutphin PD, Yen SE, Giaccia AJ. Coordinate regulation of the oxygen-dependent degradation domains of hypoxia-inducible factor 1 alpha. Mol Cell Biol. 2005;25(15):6415-6426.

41. Kaelin WG, Ratcliffe PJ. Oxygen sensing by metazoans: the central role of the HIF hydroxylase pathway. Mol Cell. 2008;30(4):393-402.

42. Piret JP, Mottet D, Raes M, Michiels C. $\mathrm{CoCl}_{2}$, a chemical inducer of hypoxia-inducible factor-1, and hypoxia reduce apoptotic cell death in hepatoma cell line HepG2. Ann N Y Acad Sci. 2002;973:443-447.

43. Koukourakis MI. Radiation damage and radioprotectants: new concepts in the era of molecular medicine. Br J Radiol. 2012;85(1012):313-330.

44. Agani F, Semenza GL. Mersalyl is a novel inducer of vascular endothelial growth factor gene expression and hypoxia-inducible factor 1 activity. Mol Pharmacol. 1998;54(5):749-754.

45. Cho $\mathrm{H}$, et al. Baicalein induces functional hypoxia-inducible factor-1alpha and angiogenesis. Mol Pharmacol. 2008;74(1):70-81.

46. Li QF, Wang XR, Yang YW, Su DS. Upregulation of hypoxia inducible factor 1alpha by isoflurane in Hep3B cells. Anesthesiology. 2006;105(6):1211-1219.

47. Lawrance IC, Fiocchi C, Chakravarti S. Ulcerative colitis and Crohn's disease: distinctive gene expression profiles and novel susceptibility candidate genes. Hum Mol Genet. 2001;10(5):445-456.

48. Giatromanolaki A, et al. Hypoxia inducible factor 1alpha and 2alpha overexpression in inflammatory bowel disease. J Clin Pathol. 2003;56(3):209-213.

49. Okuda T, et al. Hypoxia-inducible factor 1 alpha and vascular endothelial growth factor overexpression in ischemic colitis. World J Gastroenterol. 2005;11(10):1535-1539.

50. Komarov PG, et al. A chemical inhibitor of p53 that protects mice from the side effects of cancer therapy. Science. 1999;285(5434):1733-1737.

51. Brady CA, et al. Distinct p53 transcriptional programs dictate acute DNA-damage responses and tumor suppression. Cell. 2011;145(4):571-583.

52. Li T, et al. Tumor suppression in the absence of p53-mediated cell-cycle arrest, apoptosis, and senescence. Cell. 2012;149(6):1269-1283.

53. Lee CL, et al. Acute DNA damage activates the tumour suppressor p53 to promote radiationinduced lymphoma. Nat Commun. 2015;6:8477.

54. Komarova EA, et al. Dual effect of p53 on radiation sensitivity in vivo: $\mathrm{p} 53$ promotes hematopoietic injury, but protects from gastro-intestinal syndrome in mice. Oncogene. 2004;23(19):3265-3271. 55. Kirsch DG, et al. p53 controls radiation-induced 
gastrointestinal syndrome in mice independent of apoptosis. Science. 2010;327(5965):593-596.

56. Sánchez-Puig N, Veprintsev DB, Fersht AR. Binding of natively unfolded HIF-1alpha ODD domain to p53. Mol Cell. 2005;17(1):11-21.

57. El-Deiry WS. The role of p53 in chemosensitivity and radiosensitivity. Oncogene. 2003;22(47):7486-7495.

58. Leibowitz BJ, Qiu W, Liu H, Cheng T, Zhang L, Yu J. Uncoupling p53 functions in radiation-induced intestinal damage via PUMA and $\mathrm{p} 21$. Mol Cancer Res. 2011;9(5):616-625.

59. Wang X, et al. Pharmacologically blocking p53-dependent apoptosis protects intestinal stem cells and mice from radiation. Sci Rep. 2015;5:8566.

60. Qiu W, et al. PUMA regulates intestinal progenitor cell radiosensitivity and gastrointestinal syndrome. Cell Stem Cell. 2008;2(6):576-583.

61. Rezvani M, Hopewell JW, Robbins ME. Initiation of non-neoplastic late effects: the role of endothelium and connective tissue. Stem Cells. 1995;13(Suppl 1):248-256.

62. Paris F, et al. Endothelial apoptosis as the primary lesion initiating intestinal radiation damage in mice. Science. 2001;293(5528):293-297.

63. Louis NA, Hamilton KE, Kong T, Colgan SP. HIFdependent induction of apical CD55 coordinates epithelial clearance of neutrophils. FASEB J. 2005;19(8):950-959.

64. Hayden MS, West AP, Ghosh S. NF-kappaB and the immune response. Oncogene. 2006;25(51):6758-6780.

65. Cumming G, Fidler F, Vaux DL. Error bars in experimental biology. JCell Biol. 2007;177(1):7-11.

66. Rius J, et al. NF-kappaB links innate immunity to the hypoxic response through transcriptional regulation of HIF-1alpha. Nature. 2008;453(7196):807-811.

67. Gurova KV, et al. Small molecules that reactivate p53 in renal cell carcinoma reveal a NF-kappaB-dependent mechanism of p53 suppression in tumors. Proc Natl Acad Sci U S A. 2005;102(48):17448-17453.

68. Karin M. Nuclear factor-kappaB in cancer development and progression. Nature. 2006;441(7092):431-436.

69. Pasare C, Medzhitov R. Toll-like receptors: linking innate and adaptive immunity. Adv Exp Med Biol. 2005;560:11-18.

70. Rakoff-Nahoum S, Paglino J, Eslami-Varzaneh F, Edberg S, Medzhitov R. Recognition of commen- sal microflora by toll-like receptors is required for intestinal homeostasis. Cell. 2004;118(2):229-241.

71. Tallant T, Deb A, Kar N, Lupica J, de Veer MJ, DiDonato JA. Flagellin acting via TLR5 is the major activator of key signaling pathways leading to NF-kappa B and proinflammatory gene program activation in intestinal epithelial cells. $B M C$ Microbiol. 2004;4:33.

72. Burdelya LG, et al. An agonist of toll-like receptor 5 has radioprotective activity in mouse and primate models. Science. 2008;320(5873):226-230.

73. Barker N, et al. Identification of stem cells in small intestine and colon by marker gene Lgr5. Nature. 2007;449(7165):1003-1007.

74. Sangiorgi E, Capecchi MR. Bmil is expressed in vivo in intestinal stem cells. Nat Genet. 2008;40(7):915-920.

75. Yan KS, et al. The intestinal stem cell markers Bmi1 and Lgr5 identify two functionally distinct populations. Proc Natl Acad Sci U S A. 2012;109(2):466-471.

76. Metcalfe C, Kljavin NM, Ybarra R, de Sauvage FJ. $\mathrm{Lgr}^{+}$stem cells are indispensable for radiationinduced intestinal regeneration. Cell Stem Cell. 2014;14(2):149-159.

77. Paris F, et al. Endothelial apoptosis as the primary lesion initiating intestinal radiation damage in mice. Science. 2001;293(5528):293-297.

78. Moeller BJ, et al. Pleiotropic effects of HIF-1 blockade on tumor radiosensitivity. Cancer Cell. 2005;8(2):99-110.

79. Moeller BJ, Dewhirst MW. HIF-1 and tumour radiosensitivity. Br JCancer. 2006;95(1):1-5.

80. Kim WY, Oh SH, Woo JK, Hong WK, Lee HY. Targeting heat shock protein 90 overrides the resistance of lung cancer cells by blocking radiation-induced stabilization of hypoxia-inducible factor-1alpha. Cancer Res. 2009;69(4):1624-1632.

81. Moeller BJ, Cao Y, Li CY, Dewhirst MW. Radiation activates HIF-1 to regulate vascular radiosensitivity in tumors: role of reoxygenation, free radicals, and stress granules. Cancer Cell. 2004;5(5):429-441.

82. Harada H, et al. Cancer cells that survive radiation therapy acquire HIF-1 activity and translocate towards tumour blood vessels. Nat Commun. 2012;3:783.

83. Williams KJ, et al. Enhanced response to radiotherapy in tumours deficient in the function of hypoxia-inducible factor-1. Radiother Oncol. 2005;75(1):89-98.

84. Aebersold DM, et al. Expression of hypoxia-induc- ible factor-1alpha: a novel predictive and prognostic parameter in the radiotherapy of oropharyngeal cancer. Cancer Res. 2001;61(7):2911-2916.

85. Bussink J, Kaanders JH, van der Kogel AJ. Tumor hypoxia at the micro-regional level: clinical relevance and predictive value of exogenous and endogenous hypoxic cell markers. Radiother Oncol. 2003;67(1):3-15.

86. Moon EJ, Brizel DM, Chi JT, Dewhirst MW. The potential role of intrinsic hypoxia markers as prognostic variables in cancer. Antioxid Redox Signal. 2007;9(8):1237-1294.

87. Bertout JA, et al. HIF2alpha inhibition promotes p53 pathway activity, tumor cell death, and radiation responses. Proc Natl Acad Sci USA. 2009;106(34):14391-14396.

88. Pugh CW, Ratcliffe PJ. Regulation of angiogenesis by hypoxia: role of the HIF system. Nat Med. 2003;9(6):677-684.

89. Bertout JA, Patel SA, Simon MC. The impact of $\mathrm{O}_{2}$ availability on human cancer. Nat Rev Cancer. 2008;8(12):967-975.

90. Mazzone M, et al. Heterozygous deficiency of PHD2 restores tumor oxygenation and inhibits metastasis via endothelial normalization. Cell. 2009;136(5):839-851.

91. Leite de Oliveira R, et al. Gene-targeting of Phd2 improves tumor response to chemotherapy and prevents side-toxicity. Cancer Cell. 2012;22(2):263-277.

92. Williams JP, et al. Animal models for medical countermeasures to radiation exposure. Radiat Res. 2010;173(4):557-578.

93. Moding EJ, Kastan MB, Kirsch DG. Strategies for optimizing the response of cancer and normal tissues to radiation. Nat Rev Drug Discov. 2013;12(7):526-542.

94. National Institutes of Health. ClinicalTrials.gov. https://clinicaltrials.gov/ct2/results?term= Proline+hydroxylase $\&$ Search $=$ Search. cited Accessed: June 23, 2016.

95. Giaccia AJ. Molecular radiobiology: the state of the art. JClin Oncol. 2014;32(26):2871-2878.

96. Calvani M, Rapisarda A, Uranchimeg B, Shoemaker RH, Melillo G. Hypoxic induction of an HIF-1alpha-dependent bFGF autocrine loop drives angiogenesis in human endothelial cells. Blood. 2006;107(7):2705-2712.

97. Cummins EP, et al. Prolyl hydroxylase-1 negatively regulates IkappaB kinase-beta, giving insight into hypoxia-induced NFkappaB activity. Proc Natl Acad Sci U S A. 2006;103(48):18154-18159. 